\title{
Small airway epithelial-C/EBP $\beta$ is increased in patients with advanced COPD
}

\author{
Michiko Mori ${ }^{1}$, Leif Bjermer ${ }^{2}$, Jonas S. Erjefält ${ }^{1,2}$, Martin R. Stampfli ${ }^{3,4}$ and Abraham B. Roos ${ }^{2,3^{*}}$
}

\begin{abstract}
The expression of CCAAT/enhancer-binding protein (C/EBP) $\beta$ in the small airway epithelium of COPD is unknown. C/EBP $\beta$ was assessed in peripheral lung tissue of non-smoking/smoking controls and patients with GOLD I-IV COPD by quantitative immunohistochemistry. The expression of C/EBP $\beta$ was decreased in smokers compared to never smokers. Furthermore, C/EBP $\beta$ was significantly elevated in advanced COPD vs. asymptomatic smokers, and the expression correlated to lung function decline. As C/EBP $\beta$ exerts pro-inflammatory effects in the context of cigarette smoke, the elevated C/EBP $\beta$ in advanced COPD may be an indication of a breakdown of regulatory mechanisms and excessive inflammation.
\end{abstract}

Keywords: COPD, C/EBP $\beta$, Airway epithelium

\section{Findings}

Chronic obstructive pulmonary disease (COPD) is characterized by small airway inflammation. While glucocorticoids (GCs) and $\beta_{2}$ agonists are mainstay in the management of COPD, these classes of drugs are, by and large, ineffective in preventing disease progression [1]. The lack of efficient pharmaceutical options is in part due to the incomplete understanding of the intricate molecular mechanisms contributing to the disease.

The transcription factor CCAAT/enhancer binding protein $(\mathrm{C} / \mathrm{EBP}) \beta$ regulates inflammatory [2] and host defense genes [3] in the airway epithelium. Lung epithelial-C/ EBP $\beta$ activates the inflammatory response to cigarette smoke [4], as well as lipopolysaccharide (LPS) [3]. Suppression of LPS-induced airway inflammation by $\beta_{2}$ agonists is, however, also mediated by lung epithelial-C/EBP $\beta$ [3]. In addition, glucocorticoids increase the expression and transcriptional activity of $\mathrm{C} / \mathrm{EBP} \beta$. Transactivation by glucocorticoids has in contrast to pro-inflammatory stimuli been suggested to up-regulate host defense genes $[5,6]$. Hence, cigarette smoke and microbial ligands, as well as GCs and $\beta_{2}$ agonists may all activate airwayepithelial C/EBP $\beta$ in COPD, with the possibility of

\footnotetext{
* Correspondence: abraham.roos@gmail.com

${ }^{2}$ Department of Respiratory Medicine and Allergology, Lund University, Lund, Sweden

${ }^{3}$ Department of Pathology and Molecular Medicine, McMaster University,

MDCL 4084, 1280 Main Street West, Hamilton, ONL8S 4P1Canada

Full list of author information is available at the end of the article
}

different outcomes depending on the stimuli. There is currently insufficient knowledge of the expression of $\mathrm{C} / \mathrm{EBP} \beta$ in the small airways of COPD, in particular in end-stage disease where $\mathrm{GC} / \beta_{2}$ agonist therapy is mainstay.

We obtained peripheral tissue specimens from patients with stable GOLD I-IV COPD $(n=30)$, as well as controls with or without a smoking history $(n=14)$ (Table 1$)$. The study was approved by the Swedish Research Ethics Committee in Lund, Sweden. All study subjects signed informed consent to participate. Formalin-fixed and paraffin-embedded tissue sections were pre-treated with a pH 6.1 buffer (EnVision ${ }^{\text {nu }}$ FLEX Target Retrieval Solution, Dako, Glostrup, Denmark). The expression of C/EBP $\beta$ was visualized by immunohistochemistry using a polyclonal rabbit anti-C/EBP $\beta$ antibody (Santa Cruz Biotechnology, Santa Cruz, CA, USA), and EnVision ${ }^{\text {Tx }}$ Peroxidase/ DAB Detection System kit on an Autostainer Plus (DakoCytomation, Glostrup). Automated immunohistochemistry allowed for minimized operator error between tissue samples.

\section{C/EBP $\beta$ is decreased in the small airway epithelium of asymptomatic smokers}

Strong immunoreactivity to $\mathrm{C} / \mathrm{EBP} \beta$ was observed in the peripheral airway epithelium, as well as in alveolar macrophages of COPD patients and asymptomatic controls (Fig. 1a-c). C/EBP $\beta$ positive cells were furthermore identified within and in the epithelial interface of lymphoid 
Table 1 Baseline demographics and clinical characteristics

\begin{tabular}{|c|c|c|c|c|c|}
\hline Parameter & Never smokers & Smokers w/o COPD & GOLD I-II COPD ${ }^{b}$ & GOLD III-IV COPD ${ }^{c}$ & $p$ ANOVA \\
\hline Subjects $(n)^{a}$ & 8 & 6 & 18 & 12 & \\
\hline Gender (female/male) & $6 / 2$ & $3 / 3$ & $5 / 13$ & $6 / 6$ & \\
\hline Age (years) & $63 \pm 4.8$ & $56 \pm 3.2$ & $68 \pm 1.8$ & $61 \pm 1.2$ & $<0.05$ \\
\hline Height (m) & $1,64 \pm 0.033$ & $1.72 \pm 0.05$ & $1,74 \pm 0.02$ & $1,7 \pm 0.031$ & ns \\
\hline Weight (kg) & $64,6 \pm 4.6$ & $69.2 \pm 4.4$ & $73,1 \pm 3.5$ & $67,7 \pm 4.1$ & ns \\
\hline Body mass index & $23,9 \pm 1.3$ & $23.3 \pm 1.1$ & $24,4 \pm 1.1$ & $23,3 \pm 0.94$ & ns \\
\hline Pack years & N/A & $43 \pm 9.7$ & $45 \pm 3.5$ & $41 \pm 3.2$ & ns \\
\hline Smoker/ex-smoker & N/A & $3 / 3$ & $7 / 11$ & $0 / 12$ & \\
\hline FEV1/FVC & $85,9 \pm 5.7$ & $77.8 \pm 2.4$ & $61,4 \pm 1.9$ & $33,4 \pm 2.1$ & $<0.001$ \\
\hline FEV1 (\% of predicted) & $109,8 \pm 6.2$ & $93.8 \pm 4.2$ & $74,1 \pm 2.7$ & $26,2 \pm 2.7$ & $<0.001$ \\
\hline Corticosteroids (yes/no/unknown) & $0 / 8 / 0$ & 0/6/0 & $2 / 16 / 0$ & $9 / 2 / 1$ & \\
\hline Bronchodialator (yes/no/unknown) & $0 / 8 / 0$ & $0 / 6 / 0$ & $6 / 12 / 0$ & $9 / 2 / 1$ & \\
\hline
\end{tabular}

${ }^{a}$ All surgeries were performed at Skåne University Hospital, in Lund, Sweden

${ }^{\mathrm{b}}$ Tissue samples were obtained during lung resection surgery for bronchial tumour

${ }^{\mathrm{C}}$ Tissue samples were obtained from GOLD II COPD patients during lung resection surgery for bronchial tumour, and from GOLD IV COPD patients during

lung transplantation

follicles [7], in lung tissue collected from patients with COPD (inlet of Fig. 1c).

An Aperio ScanScope Slide Scanner (Aperio Technologies, Vista, CA) was used to generate digital images of the tissue sections, and morphometric analyses were performed using Aperio ImageScope v.10.0 software (Aperio Technologies) [8]. Computerized image analysis revealed that the expression of $\mathrm{C} / \mathrm{EBP} \beta$ was significantly lower in the airway epithelium among asymptomatic controls with a smoking history compared to never

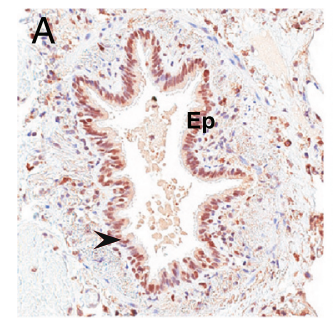

D
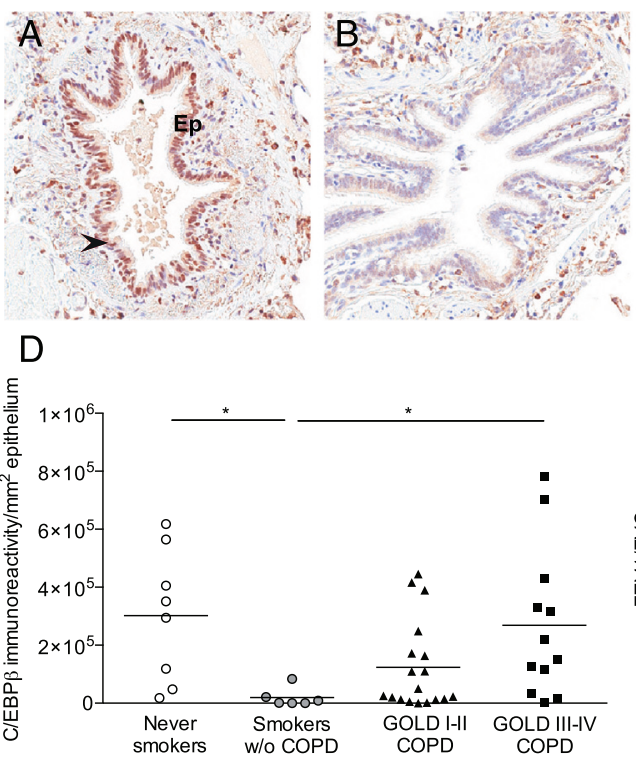

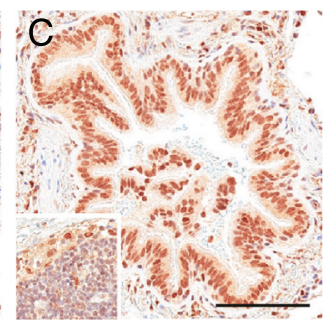

E

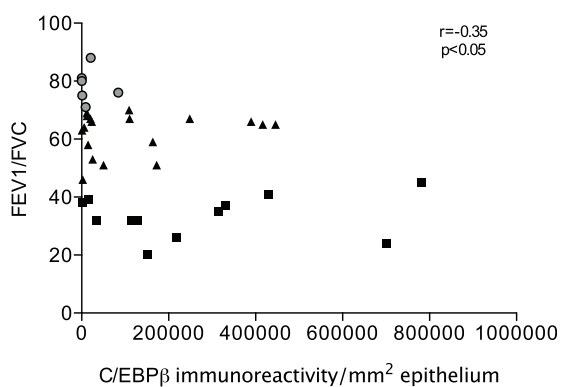

Fig. 1 Reduced expression of C/EBP $\beta$ in asymptomatic smokers and elevated expression in advanced COPD. Light micrographs of the immunoreactivity to CCAAT/enhancer-binding protein (C/EBP) $\beta$ in peripheral pulmonary tissue of a (a) never-smoker, (b) asymptomatic smoker, and (c) patient with very severe chronic obstructive pulmonary disease (COPD). Immunoreactivity was detected by DAB (brown). Sections were counterstained with Mayer's Hematoxylin (blue). Scale bar indicates $100 \mu \mathrm{m}$. The epithelium (Ep) is denoted and an arrowhead indicates a positive cell in (a). Inlet of (c) shows immunoreactivity to C/EBP $\beta$ in a tertiary lymphoid follicle. $\mathbf{d}$ Immunoreactivity (defined as number of positive pixels $/ \mathrm{mm}^{2}$ ) to C/EBP $\beta$ in peripheral lung epithelium of never-smokers, asymptomatic smokers and patients with mild-moderate (global initiative of COPD, GOLD I-II) and severe-very severe (GOLD III-IV) COPD. Horizontal lines indicate mean value. e Pearson correlation coefficient analysis of the immunoreactivity $/ \mathrm{mm}^{2}$ to C/EBP $\beta$ in peripheral lung epithelium and the forced expiratory volume in 1 second (FEV1)/forced vital capacity (FVC) of smokers without COPD and patients with GOLD I-IV COPD. Clear circles: never smokers without airway obstruction; grey circles: asymptomatic smokers, triangles: GOLD I-II COPD; squares: GOLD III-IV COPD. $n=6-18 .{ }^{*} p<0.05$ 
smokers $(p<0.05$, Fig. 1d). The lower expression of $\mathrm{C} /$ EBP $\beta$ was associated with a reduced immunoreactivity to the Marker of Proliferation (M) KI67 (rabbit polyclonal antibody A0047, DakoCytomation) suggestive of an attenuated proliferation of the airway epithelium $(0.02 \pm 0.004$ vs. $0.0082 \pm 0.0026$; mean \pm SEM, $p<0.01)$.

The reduced expression of $\mathrm{C} / \mathrm{EBP} \beta$ corroborates our previous finding of significantly decreased $C E B P B$ mRNA in the bronchial epithelium of current and former smokers, compared to never smokers [4], and reduction of $C E B P B$ mRNA and C/EBP $\beta$ protein in bronchial epithelial cells stimulated with cigarette smoke extract in vitro [4]. Thus, $\mathrm{C} / \mathrm{EBP} \beta$ is down-regulated by cigarette smoke in both the proximal and distal airway epithelium. This may be part of a compensatory mechanism of feed back inhibition, as an adaptive attempt to control chronic inflammation. $\mathrm{C} / \mathrm{EBP} \beta$ contributes to the differentiation of the airway epithelium during organogenesis, and promotes club cell differentiation at the expense of goblet cell differentiation [9]. As cigarette smoke stimulates goblet cell differentiation in vitro [10], decreased expression of $\mathrm{C} / \mathrm{EBP} \beta$ in the distal airways may thus provide a mechanistic explanation for goblet cell hyperplasia induced by cigarette smoke. While the smokers included in our study were asymptomatic, decreased $C / E B P \beta$ may over time lead to clinical presentation with mucus hypersecretion. In support of this, the activity of C/EBP $\beta$ in the bronchial epithelium is decreased in smokers with chronic bronchitis [11], compared to asymptomatic smokers.

\section{Airway epithelial-C/EBP $\beta$ is elevated in advanced COPD}

The expression of airway epithelial-C/EBP $\beta$ was significantly increased in advanced (GOLD III-IV) COPD, compared to asymptomatic smokers $(p<0.05$, Fig. 1d). Furthermore, a negative correlation between lung function and the airway expression of $C / E B P \beta$ was observed $(r=-0.35 p<0.05$, Fig. 1e), suggesting a role for C/EBP $\beta$ in disease progression. The expression of the lungenriched $\mathrm{C} / \mathrm{EBP} \alpha$, which cooperates with $\mathrm{C} / \mathrm{EBP} \beta$ in various cellular functions [2], was not significantly different in any of the groups within the cohort $(\mathrm{C} / \mathrm{EBP} \alpha$ rabbit polyclonal antibody (14AA) sc-61, Santa Cruz Biotechnology, Dallas, TX, USA; data not shown).

Mechanistically, it is possible that the elevation of $\mathrm{C} /$ EBP $\beta$ represents a breakdown of the suggested feed back inhibition observed in cigarette smoke-induced inflammation, leading to escalating inflammatory processes in endstage COPD. Alternatively, chronic bacterial colonization among COPD patients [12] may activate C/EBP $\beta$. It is, however, also possible that steroid and $\beta_{2}$ agonist treatment effected the expression of C/EBP $\beta$ in our study, as airway epithelial-C/EBP $\beta$ is induced/activated by GCs and $\beta_{2}$ agonists $[3,6]$. This may represent a novel mechanism by which GCs and $\beta 2$ agonists modulate the transcriptional profile of the airway epithelium in advanced COPD. Future studies should address whether the elevation of $C / E B P \beta$ is disease- or treatment-specific, and if GCs and $\beta_{2}$ agonists induces C/EBP $\beta$ to promote hostdefenses and act anti-inflammatory, or pro-inflammatory.

\section{Competing interests}

The authors declare that they have no competing interests.

\section{Authors' contributions}

MM, LB, JSE, MRS and ABR conceived of and designed the study. MM and ABR performed experiments. MM, JSE, MRS and ABR analyzed and interpreted data. MSR and ABR wrote the manuscript. All authors read and approved the final manuscript.

\section{Acknowledgements}

We would like to thank Britt-Marie Nilsson and Karin Jansner at Lund University, and Joanna Kasinska and Marie Bailey at McMaster University for technical support and skillful assistance.

\section{Author details}

'Department of Experimental Medical Science, Lund University, Lund, Sweden. ${ }^{2}$ Department of Respiratory Medicine and Allergology, Lund University, Lund, Sweden. ${ }^{3}$ Department of Pathology and Molecular Medicine, McMaster University, MDCL 4084, 1280 Main Street West, Hamilton, ONL8S 4P1Canada. ${ }^{4}$ Department of Medicine, Firestone Institute of Respiratory Health at St. Joseph's Health Care, Hamilton, ON, Canada.

Received: 21 April 2015 Accepted: 22 October 2015

Published online: 29 October 2015

\section{References}

1. Vestbo J, Hurd SS, Agustí AG, Jones PW, Vogelmeier C, Anzueto A, et al. Global Strategy for the Diagnosis, Management, and Prevention of Chronic Obstructive Pulmonary Disease. Am J Respir Crit Care Med. 2013;187:347-65.

2. Cassel TN, Nord M. C/EBP transcription factors in the lung epithelium. Am J Physiol Lung Cell Mol Physiol. 2003;285:L773-781.

3. Roos AB, Barton JL, Miller-Larsson A, Dahlberg B, Berg T, Didon L, et al. Lung epithelial-C/EBPbeta contributes to LPS-induced inflammation and its suppression by formoterol. Biochem Biophys Res Commun. 2012;423:134-9.

4. Didon L, Barton JL, Roos AB, Gaschler GJ, Bauer CM, Berg T, et al. Lung epithelial CCAAT/enhancer-binding protein-beta is necessary for the integrity of inflammatory responses to cigarette smoke. Am J Respir Crit Care Med. 2011;184:233-42.

5. Zhang N, Truong-Tran QA, Tancowny B, Harris KE, Schleimer RP. Glucocorticoids Enhance or Spare Innate Immunity: Effects in Airway Epithelium Are Mediated by CCAAT/Enhancer Binding Proteins. J Immunol. 2007;179:578-89.

6. Berg T, Cassel TN, Schwarze PE, Nord M. Glucocorticoids regulate the CCSP and CYP2B1 promoters via C/EBPbeta and delta in lung cells. Biochem Biophys Res Commun. 2002:293:907-12.

7. Mori M, Andersson CK, Svedberg KA, Glader P, Bergqvist A, Shikhagaie M, et al. Appearance of remodelled and dendritic cell-rich alveolar-lymphoid interfaces provides a structural basis for increased alveolar antigen uptake in chronic obstructive pulmonary disease. Thorax. 2013;68:521-31.

8. Roos AB, Mori M, Gronneberg R, Osterlund C, Claesson HE, Wahlstrom J, et al. Elevated exhaled nitric oxide in allergen-provoked asthma is associated with airway epithelial iNOS. PLoS One. 2014;9:e90018.

9. Roos AB, Berg T, Barton JL, Didon L, Nord M. Airway epithelial cell differentiation during lung organogenesis requires C/EBPalpha and C/EBPbeta. Dev Dyn. 2012;241:911-23.

10. Haswell LE, Hewitt K, Thorne D, Richter A, Gaça MD. Cigarette smoke total particulate matter increases mucous secreting cell numbers in vitro: $A$ potential model of goblet cell hyperplasia. Toxicol In Vitro. 2010;24:981-7.

11. Didon L, Qvarfordt I, Andersson O, Nord M, Riise GC. Decreased CCAAT/ Enhancer Binding Protein Transcription Factor Activity in Chronic Bronchitis and COPD. Chest. 2005;127:1341-6.

12. Murphy TF, Brauer AL, Schiffmacher AT, Sethi S. Persistent colonization by Haemophilus influenzae in chronic obstructive pulmonary disease. Am J Respir Crit Care Med. 2004;170:266-72. 